Recepción: 30/10/2018

Evaluación: 31/10/2018

Aprobación: 06/11/2018

Artículo de Investigación-Reflexión

DOI: https://doi.org/10.22267/rhec.192222.57

\title{
Economía de la educación: un análisis dialógico
}

\author{
Ana Isabel Suárez Martínez ${ }^{1}$ \\ Colegio Cooperativo Monseñor Ismael Perdomo, Colombia \\ Diego Andrés Fernández ${ }^{2}$ \\ Institución Educativa Distrital Bosanova, Colombia
}

\section{Resumen}

Este artículo presenta un análisis sobre la realidad de la educación en América Latina, enfatizando aspectos económicos de afectación directa sobre todas sus dinámicas. Se pretende, entonces, evidenciar cómo, en esa interacción, se desvirtúa el objetivo primordial de la educación a favor de intereses economicistas y cómo el sentido de la escuela se ha tergiversado a merced de la industria, lo que propende por una dicotomía polivalente en el que la concepción de capital humano cada vez es más cercana a los fines mercantilistas propuestos por las agencias internacionales.

Palabras clave: Administración de la Educación; Eficiencia de la Educación; Reforma de la Educación.

\footnotetext{
1 Licenciada en Educación Básica con énfasis en Ciencias Sociales. Docente en Colegio Cooperativo Monseñor Ismael Perdomo. Correo electrónico: Isasu01@hotmail.com. Código ORCID: https://orcid.org/0000-0002-4473-5207

2 Doctor en Educación. Docente en Institución Educativa Distrital Bosanova. Correo electrónico: diegoandresf@gmail.com. Código ORCID: https://orcid.org/0000-0001-7204-8976
} 


\title{
Economics of education: a dialogical analysis
}

\begin{abstract}
This article presents an analysis of the reality of education in Latin America, emphasizing economic aspects that directly affect all its dynamics. The aim is to show how this interaction misrepresents the primary objective of education in favor of economic interests and how the meaning of school has been distorted at the mercy of industry, tending towards a multipurpose dichotomy in which the conception of human capital is increasingly closer to the mercantilist purposes proposed by international agencies.
\end{abstract}

Keywords: Education Administration; Education Efficiency; Education Reform.

\section{Economia da educação: uma análise dialógica}

\section{Resumo}

Este artigo apresenta uma análise da realidade da educação na América Latina, enfatizando aspectos econômicos de impacto direto em toda a sua dinâmica. Pretende-se, então, mostrar como, nessa interação, se desvirtua principal objetivo da educação em favor dos interesses económicos e como o sentido da escola foi deturpado à mercê da indústria, propiciando uma dicotomia polivalente em que a concepção de capital humano Está cada vez mais próximo dos propósitos mercantilistas propostos por agências internacionais.

Palavras-chave: administração educacional; Eficiência na educação; Reforma da educação. 


\section{Introducción}

La educación se ha mencionado, a lo largo de la Historia, como un componente de la humanidad articulado transversalmente a la problemática economicista; es, sin duda, uno de los espacios trabajados y reflexionados intensamente durante décadas desde diferentes ciencias, que dan sus aproximaciones a diversas premisas o asuntos. Una de esas premisas, legitimada dentro de los sistemas de gobierno, como el democrático, es: la educación como derecho fundamental, que pretende, de manera equitativa, el acceso en todos sus niveles. Este acceso, a través de los años, ha estado permeado por diversos debates, como alfabetizar y escolarizar, o el llegar a comunidades necesitadas, o a la que se asiste con mayor control: la administración financiera de ese acceso.

Precisamente, este artículo se propone exponer este aspecto, que encierra cuestionamientos que contrarían el valor propio de este derecho, pues se ha tergiversado, desde una mirada empresarial reduccionista de la educación, hacia conceptos económicos. En este sentido, siguiendo a Tomasevski (2004), se puede decir que no se puede ligar el desarrollo como la elaboración de derechos humanos, porque ese desarrollo considera tajantemente a las personas como capital humano. En efecto, si los seres humanos son un objeto de inversión, se los convierte en medios, antes que en fines del desarrollo.

La fórmula utilizada para pensar la educación desde esta mirada es la formación igual a la productividad y ésta igual a mayores ingresos; en consecuencia, se da por hecho una directa proporcionalidad entre la productividad y los ingresos. De esta manera, se mecaniza una producción exitosa del proceso educativo materializado en el aprendizaje para la producción; por eso, tiende a pensarse en función de esta, como sinónimo de calidad.

\section{2. ¿La economía de la educación, sinónimo de desarrollo?}

En efecto, el fenómeno educativo articulado a las lides económicas tiene una mirada envuelta en una supuesta racionalidad, conocida como la "economía de la educación", que, en lo fundamental, maneja la teoría de capital humano, acuñada por economistas estadounidenses a mediados del siglo XX, que opera dentro del entramado teórico a través de una red de conceptos explicativos en torno a una 
serie de fórmulas descriptivas, que prescriben un carácter económico de producción clásica, con tintes políticos, lo que, según un determinado tipo de gobierno, puede representar, en conveniencia, una inversión formativa para sus ciudadanos.

Se conjuga en unas categorías clásicas económicas, como la maquinaria, el trabajo y el capital; de este último surge el capital no material, situado en las mentes de los que posibilitan la adquisición de activos físicos. Desde aquí se aborda la problemática referente al capital humano, capital que se expone o direcciona hacia un tipo de inversión; es decir, la obtención de mejores resultados con el dinero destinado al control del gasto público, para, de esta forma, generar un proceso de inversión más efectivo.

A partir de esta situación, se han desarrollado estudios empíricos acerca de la tasa de retorno de la educación, el análisis de gasto educativo, el financiamiento y su vinculación con el sector productivo, entre otros factores que generan impacto en el crecimiento económico de diversos países, tal como lo fue, en sus inicios, en el caso anglosajón, posteriormente en Europa continental y recientemente en el pueblo latinoamericano. Se podría suponer, a partir de lo expuesto, que la Economía no es ajena a los problemas políticos, especialmente cuando se habla de Estado y, menos aún, podría tratarse de una ciencia neutral, pues ha direccionado cambios sustanciales en la formulación y puesta en marcha de políticas públicas; en otras palabras, se podría decir que:

(...) aunque todos los niños, independientemente de su condición social, tuvieran acceso a la educación básica, es muy probable que aquellos pertenecientes a los estratos sociales más bajos presenten un desempeño más pobre con relación a los niños de estratos sociales más altos. Esto se vería reflejado en mayores índices de repitencia, deserción, menores calificaciones. (Morduchowicz 2004, 45)

Estas políticas traen consigo rasgos de desigualdad, desinterés social, racismo y una enorme brecha social, pues no se debe olvidar que esta teoría tiene como país de origen a los Estados Unidos. Externamente, en términos económicos, estas premisas son totalmente equitativas teniendo en cuenta el costo-beneficio, la rentabilidad, los filtros de alcance, la inversión-ingreso-productividad, una estratificación social y la educación como bien de consumo, entre otras cualidades, demostrables estadísticamente por la formulación de las premisas teóricas. 
En realidad, en el contexto latinoamericano, se encuentran engranajes del aparato educativo diferenciado y heterogéneo. Se responde a realidades económicas, políticas, sociales y culturales diversas, pues a su gente la han marcado procesos de violencia, discriminación, pobreza, inexistencia funcional del Estado, entre muchos otros factores que le han impreso una identidad especial.

En ese orden de ideas, Tomasevski (2004) señala que los derechos humanos obligan a preguntarse sobre el modo en que la pobreza se cruza con la discriminación; por tanto, si los niños son pobres, porque sus endeudadas familias los cedieron a los acreedores de castas superiores para pagar las deudas, entonces el origen del problema está en la discriminación basada en castas. Por otra parte, si a ellos se los excluye de la escuela, porque pertenecen a minorías marginadas, los fondos para su educación no ayudan hasta que no se supere la discriminación contra las minorías.

Al formular estas consideraciones, se puede inferir que la educación, desde esta perspectiva, se pensó, en un primer momento, como un mecanismo de producción, pero, en la actualidad, sigue teniendo un carácter meramente instrumental; en ese orden de ideas:

(...) la globalización neoliberal no sólo nos enfrenta a un desfinanciamiento en el sector de la educación, sino que, así mismo, asistimos a una individualización de los jóvenes y estudiantes. La crisis que está sufriendo el sistema capitalista, no sólo repercute en las ganancias de la hegemonía del capital, sino que también se introduce en los individuos de la sociedad, quienes han caído en la lógica egoísta y ensimismada; aquí cada quien lucha por sobrevivir y "triunfar", por alcanzar el éxito sin importar las desmedidas formas que le acerquen a ello; el sistema ha dibujado como enemigo al otro (a); sólo importa lo mío y el desinterés por el mundo y lo que le rodea son segundos y hasta terceros planos; pensar en colectivo no es una opción para el capitalismo (Galindo, Gómez y Rodríguez, 2014, 88).

La consideración que se tiene de las personas es, en consecuencia, la visualización secular de actores vinculados a una estructura donde operan como piezas funcionales del engranaje productivo. Por eso, conviene recalcar sobre la importancia de repensar la educación, inmersa en un ámbito interdisciplinar que considere la relación entre educación y desarrollo, al tomar en cuenta a las personas: su realidad política, social y fundamento cultural. 
Si bien la teoría del capital humano abarca cuatro consideraciones: la educación formal, la experiencia laboral, la capacitación recibida en su trabajo, bien sea público o privado, y la salud, que influye directamente en la productividad; no incluye perspectivas de suma relevancia, que han contribuido a la reflexión sobre educación, como la pedagogía, los maestros y la escuela. Por esta razón, tal como lo señalan Martínez Rodríguez y Amador Muñoz, "es preciso conjugar crecimiento económico con desarrollo social y político, por lo que la educación no va a tener exclusivamente una función instrumental orientada también a sus efectos sobre la autoestima, autorrealización y la libertad de las personas". $(2010,89)$

Toda persona tiene la oportunidad de labrarse condiciones dignas a través de la educación, elemento que necesita transcender la práctica instructiva del aula para materializarse en una formación que propiciase cambios en la realidad socioeconómica actual. Sin embargo, es necesario recalcar que no es del todo negativo que los economistas se acerquen al escenario educativo y generen aportes desde una perspectiva cuantitativa, aspectos que otras ciencias no ven o estudian, pero cuando estos aportes enfatizan sus estudios en el reducido énfasis economicista, ocasionan la disminución de perspectivas interdisciplinares, que dan por resultado una sola explicación a los múltiples asuntos educativos, para producir, de esta manera, imaginarios negativos, que sesgan principalmente la función de los componentes más importantes en el acto educativo: el maestro y los estudiantes.

Al seguir esta misma tendencia, se puede afirmar que:

(...) estudios de problemas sociales de los conglomerados urbanos, de las estructuras industriales y de las redes de servicios, proveen a los responsables de este escenario particular por la toma de decisiones en materia de política social $-\mathrm{y}$ de política educativa - de elementos sólidos para sistematizar la selección de objetivos y la evaluación económica de los programas correspondientes. ( Kleiman, 2000, 165)

Una mirada compleja del fenómeno educativo, no necesariamente ininteligible, considera dinámicas culturales, históricas y, por tanto, imposibles de aprehenderse de manera exclusiva por variables económicas; sería un problema pretender que se basara la toma de decisiones derivadas de la política educativa en estudios que tienen una forma fragmentada de entender la escuela, el aprendizaje, la enseñanza y, en particular, la función de los maestros. 


\section{El predominio neoliberal en la educación}

Precisamente, al referirse al criterio económico, político y social que predomina, el neoliberalismo se inserta en la realidad, que se ve abocada a las tres características: competencia, eficiencia y eficacia, con la inserción internacional. Se debe tener en cuenta que cada estilo de desarrollo genera, también, un estilo educativo, que no solo se refiere a los recursos del sector, sino, además, a cómo se piensan la función de la educación, el ideal educativo y las políticas pedagógicas.

Recuérdese que la educación es uno de los componentes más importantes para el desarrollo económico; en este sentido, el estilo que se adoptara va a mostrar un camino, una dirección en materia social, económica y política. Precisamente, el modelo neoliberal se establece en la búsqueda permanente de recursos que no se devenguen del Estado, sino del sector privado, recursos no solamente monetarios, sino humanos: mano de obra eficiente adecuada a los servicios y los tipos de producción que se privilegien. En este caso, un modelo educativo da por sentado un ideal educativo.

Este es un ideal educativo de carácter competitivo, para alcanzar la excelencia por medio de las individualidades sobre lo colectivo; se busca, entonces, el progreso por medio de la inversión. En sí, una educación desprovista de carácter solidario y social, una totalmente excluyente y vivaz, que no presta atención a las necesidades contextuales de la región latina, reclamante de medidas políticas y económicas para superar las graves falencias de financiación para la educación.

Aunque se enaltece la cobertura educativa —en su mayoría primaria- en muchos de estos países, no es suficiente para que pudiera competir con los estándares de países desarrollados. No es raro afirmar que se requiere con urgencia la participación educativa para una cobertura en niveles secundarios y universitarios, en países marcados por la desigualdad y la pobreza; en ese orden de ideas:

(...) Pese a los sólidos argumentos económicos y no económicos que justifican incrementos en la inversión educativa, la mayor parte de los países de la región encuentra dificultades para obtener recursos adicionales para el sector. Se observa que los más desarrollados dedican una mayor proporción de su riqueza a la inversión en educación y cambio tecnológico, esfuerzo que se refleja en mayores niveles de escolarización alcanzados por la población adulta (Morduchowicz y Duro, 2007, 25). 
El gasto público ha sido uno de los principales mecanismos para evitar situaciones de miseria, pero, durante los procesos de ajuste, perdió gran parte de esa función; en ello incidieron, sobre todo, dos factores: por una parte, para lograr el equilibrio fiscal global, se actuó respecto a la reducción del gasto público no financiero sobre el aumento de la recaudación; $y$, por la otra, se privilegió el pago de intereses de la deuda externa por encima de los demás gastos públicos. En este sentido, se reduce la inversión en el sector público y en su infraestructura; se asistió a una degradación de los recursos públicos por la carencia de inversión, lo cual lo aprovechó el sector privado para ofrecer servicios educativos; es decir, que:

La educación, eso sí, generalmente es uno de los rubros más altos dentro de los presupuestos públicos, pero dichos presupuestos están atados a políticas que limitan el gasto en pro del control del déficit fiscal. En otras palabras, la educación tiene la mayor parte de la menor parte. (Cabrera, 2007, 4).

También, lo argumentan las Naciones Unidas y la Cepal, en un reciente estudio, del 2016:

(...) Bolivia (Estado Plurinacional), el Ecuador y Panamá han aumentado fuertemente sus niveles de inversión pública, alcanzando cifras superiores a los 10 puntos porcentuales del PIB, magnitudes muy lejanas del promedio de la región. En Chile, Colombia, México y el Perú, los niveles se han mantenido cercanos al 5\% del PIB. La Argentina, Costa Rica, la República Dominicana y el Uruguay muestran magnitudes dispares. (Naciones Unidas y Cepal, 2016, 26).

Precisamente, la educación recibe financiamiento de diversas fuentes, pero el mayor porcentaje se da desde lo gubernamental; sin embargo, en los últimos años, se instauran otras fuentes de financiamiento, entre las cuales se encuentran: el sector privado, la recaudación de impuestos, los fondos presupuestales de índole interna o externa, además de la cooperación internacional bilateral y multilateral. Según esto, se pensaría que existen diferentes entradas de financiación, que mejorarían la gestión educacional, pero, en realidad, se sigue dependiendo de la fuente estatal.

Aunque existiera el fervor privado, poco realiza en cuanto a la transferencia de inversión para la educación, pero sí aprovecha el auge del gasto familiar en educación privada. Es inevitable negar que, en lo estatal, se asiste a una crisis fiscal, donde no se tiene clara la rendición de cuentas dada por entes territoriales 
- municipales-, ni mucho de los ingresos del PIB que se destinan al tema de educación, además del ajuste del gasto para evitar un déficit fiscal. En palabras de Miñana Blasco y Rodríguez,

El estado debe concentrar sus esfuerzos en programas focalizados en los sectores de pobreza extrema y una red de seguridad para situaciones coyunturales de necesidad, restaurando así la capacidad de los individuos de reingresar al mercado $(2002,9)$.

Al seguir este mismo marco de referencia, se podría afirmar que:

Si las relaciones de mercado deben primar, también el «peso» financiero del Estado se debe reducir. Los impuestos no deben distorsionar ni interferir en las asignaciones del mercado por lo que los impuestos indirectos, el de consumo en forma particular, pasan a ocupar el primer lugar de la lista de recaudadores. Por otro lado, tal concepción implica que el equilibrio fiscal se erige en un objetivo de primer orden. (Papa, 2004, 11)

Si no se piensa en revisar detenidamente el sistema de financiación y la estructura fiscal de estos países, resulta imposible pensar en la equidad, que se proclama constantemente en el discurso neoliberal; sin embargo, se manejan de manera ineficiente los recursos de toda índole, que deben invertirse en la mejora sustancial de la educación. Se le exige, pues, al Sistema educativo, una gestión eficiente para atender las demandas de una población heterogénea; estudios realizados por las Naciones Unidas y la Cepal, en 2004, señalan que la población se encuentra en la edad de asistir a la escuela primaria y secundaria y que, en algunos países, como Guatemala, Haití, Nicaragua, Honduras y Belice, la proporción de la población está en un $40 \%$.

Se necesita repensar la educación como un bien común del que la sociedad igualitariamente tuviera beneficios, donde, por la intervención del Estado, se pudiera acceder a una educación sin brechas económicas ni sociales; no obstante, esta labor masificadora se produce de la manera menos idílica posible, pues es perentorio reconocer, también, que se encuentra en predisposición con el sector privado, que abona o reviste porcentajes para la entrada de la sociedad a la educación. Estos espacios no los cubre el Estado debido a sus fallas estructurales; ahora bien, estos porcentajes de ninguna manera deben interferir con la función estatal, que debe situarse en pro del bien común y no en un bien de mercado. 
Con frecuencia, se piensa que resulta benéfica la sobre-especialización: educación como bien de mercado y no como oportunidad de formación de conocimiento, que sitúa al individuo en la capacidad de moverse ágilmente en el amplio espacio mercantilista, para proveerlo de ganancias materiales que, a la postre, la determinan como "educación de credencial", tal como es el caso de una de las piezas fundamentales en el proceso educativo: el maestro que, al seguir a Feldfeber (2007), debe moverse con agilidad en las redes de mercado, de acuerdo a unos índices de productividad, medidos por su nivel de capacitación y titulación, que le permitan el ascenso en el mundo laboral.

Se puede afirmar que el esquema investigativo del capital humano ha desplegado una feracidad realmente asombrosa, que ha generado nuevos proyectos de investigación en casi todas las ramas de la Economía. Un aspecto importante es el que se refiere a la educación para el desarrollo económico, en la que se señala una educación contributiva para la mejora del capital humano. Este aspecto es discutible, por estar estrechamente ligado a factores determinantes respecto a cómo influye y qué tipo de educación se requiere para el desarrollo de los países.

Por supuesto, Latinoamérica no ha tenido niveles óptimos de calidad, según los parámetros neoliberales, debido a los bajos resultados en pruebas internacionales y nacionales, propias de cada país, que no permiten, según la formulación neoliberal, un desarrollo anclado a una economía productiva. En este sentido, al seguir a Moura Castro e Ioschpe (2007), la educación latinoamericana, comparada con la de otras partes del mundo, y contrastada con los niveles de renta por capital, presenta niveles cualitativamente inferiores a los esperados; resulta débil, a pesar de los altos niveles de ingreso de estudiantes matriculados.

La Economía de la educación pretende dar respuestas racionalmente económicas a estos temas y los que surgen en la cotidianidad, hecho que, entre otras cosas, resulta discutible, dado que, derivado de tal pretensión, se puede inferir un enfrascamiento en la que no resulta beneficiosa para el crecimiento humano, la concepción de una política que compromete los recursos públicos y las realidades contextuales de todo un Sistema educativo y, consecuentemente, de una sociedad.

Las exigencias de eficiencia y aptitud, como características fundamentales en áreas laborales empresariales, se han trasladado de manera abrupta a la educación, al considerar nuevos objetivos: como la competencia y la competiti- 
vidad, que llevan a que la funcionalidad de la escuela se transformase; es decir, el saber que se adquiere en ella y los medios para alcanzarlo, como lo son que la enseñanza y el aprendizaje cambiasen.

En este sentido, la escuela tiene que acomodarse, para ser más funcional ante las nuevas exigencias actuales. Al insertarse como pilar en el desarrollo de una sociedad, en un contexto marcado por la economía de mercado, la educación comienza a pensarse desde las lógicas de la competitividad, la globalización y el mercado; en este sentido, llega a ser un componente de desarrollo de vital importancia para el crecimiento económico y social de los países, pero se ve reducido a un componente meramente económico, que se mercantiliza. Al seguir esta misma línea, se podría afirmar que:

El nuevo desarrollismo, en cambio, impulsa reformas institucionales que, además de fortalecer el mercado, fortalezcan el Estado, ya que solo un Estado con un aparato eficiente y con instituciones dotadas de legitimidad puede servir de instrumento a la sociedad. Para el nuevo desarrollismo, la Nación - la sociedad nacional solidaria - es el agente fundamental del desarrollo. Para lograr este desarrollo, no alcanza con garantizar la propiedad y los contratos, sino que es necesario elaborar una estrategia nacional de desarrollo que estimule a los empresarios a invertir y priorice las exportaciones y los sectores económicos con un alto valor agregado per cápita, que generen bienes intensivos en tecnología y conocimiento. ( Bresser-Pereira, 2007, 124)

Desde luego, para lograr la competitividad necesaria se requiere que se alcance calidad educativa; para esto, los procesos educativos se transforman acordes a políticas internacionales estandarizadas, en procesos que se encargarán de medir rigurosamente esa calidad, como, por ejemplo, la evaluación, que cobra una gran importancia para medir los aspectos educativos, que van desde la planificación hasta la puesta en marcha de planes evaluativos generalizados. Estos procesos se condicionan a procesos macroeconómicos de cada uno de los países por la financiación, aspecto que resulta crucial para la marcha de la eficiencia educativa y, por ende, de su calidad.

\section{La educación internacionalizada y los países que se siguen desarrollando}

Estos pilares imperantes, propios de las nuevas realidades, los marcan el mercado, la globalización, la dicotomía entre desigualdad y tecnología vanguardista, lo que supone el avance generalizado por niveles de la educación en 
cada país, sin tener en cuenta los contextos propios de los países del planeta, los que responden a necesidades inherentes a sus sistemas económicos, políticos y culturales, pero es necesario revisar en qué se piensa cuando se refiere a calidad (eficacia - eficiencia) para acceder a esa sociedad de conocimiento, que representa las auras de la globalización; en ese orden de ideas:

La mayor parte de los países de América latina han comenzado profundos e integrales procesos de transformación de sus sistemas educativos. Estos procesos tienen algunos aspectos en común. Uno de ellos se refiere al contexto. En todos los casos ocurren en escenarios rápidamente cambiantes, afectados por la emergencia de la sociedad de la información y del conocimiento, la articulación a la economía mundial y la reapertura de la oportunidad de desarrollo democrático. (Braslavsky y Acosta, 2001, 8)

La calidad, vista como obtención resultadista de los procesos educativos en diversos países - específicamente los latinoamericanos-, se limita por aspectos tales como la desigualdad, escasos recursos e infraestructura pobre, que no corresponde, en primer lugar, a la concepción educacional de derecho y, segundo, a la educación del siglo XXI. No obstante:

(...) el entramado educativo del neoliberalismo se corresponde con el capitalismo de nuestro tiempo que abjura de la educación como un derecho y lo convierte en un servicio mercantil, algo inscrito en el proceso general de mercantilización de todo lo existente, lo cual no se da solamente en los países capitalistas centrales, sino que se proyecta por el resto del planeta, a través del Consenso Educativo de Washington. (Vega Cantor, 2011, 337)

Estas problemáticas se presentan con más fuerza en países en vía de desarrollo, que son invisibles para gobiernos donde se le da prioridad al sector privado. Una sociedad mundial se proclama sociedad de conocimiento, pero no atiende a las necesidades básicas de la mayoría de la población, que forma parte precisamente de la educación pública y que no está en la lista de prioridades del ejercicio del gobierno.

Una de las problemáticas más apremiantes, y en crisis en varios países de la región, es el financiamiento de la educación. En este campo, lo público se ve amenazado por la poca injerencia de equidad del ente estatal; de esta manera, el sector privado sigue en auge y la población que depende de un cupo universitario en una universidad pública se ve en la dificultad de ingresar a ella dado el recorte de cupos por carreras. Lo público sigue siendo uno de los sectores más afectados 
por la inequidad presupuestal, al igual que los demás sectores de la sociedad que, también, viven la misma historia, al estar en medio de la disyuntiva entre cobertura y calidad; al respecto, es importante acotar que:

(...) La noción de calidad está atravesada primordialmente por las lógicas instauradas por los sistemas de gestión de tipo empresarial, siendo así que su asimilación denota aspectos de orden operativo y calificador. También se pudo apreciar una apuesta que dirige la atención hacia otra mirada de la calidad, que se desprende de las lógicas fundamentadas en la política neoliberal y se adhiere a unos presupuestos centrados en las necesidades sociales, culturales y pedagógicas que exige la sociedad actual. De esta forma la calidad debe ser entendida en la lógica de la educación y no en la lógica empresarial. (Mallama Martínez, 2014, 16)

En este mismo sentido:

(...) A la hora de mirar la región en su conjunto, es preciso pensar la trama abierta entre el neoliberalismo y el postconsenso de Washington como un lugar de disputa por los sentidos de la educación, de negociaciones de significado y de lucha cultural por definir las orientaciones político educativas, las nociones de igualdad que las sustentan, la concepción de derecho a la educación en que deben fundarse y el rol que los Estados deben asumir en este proceso. Esta lucha, que es disputa por la hegemonía, se mantiene vigente para la región. De esto dan cuenta la gran cantidad de matices que es posible identificar en un análisis comparado y, también, el modo en que significados neoliberales se conjugan con otras definiciones, incluso en aquellos países comprometidos, con mayor o menor radicalidad, en construcciones que se proponen como críticas y alternativas respecto del neoliberalismo (Saforcada y Vassiliades, 2011, 302)

Re-pensar la calidad de la educación es un reto mucho mayor y difícil de comprender cuando se detiene en la realidad, llena de desigualdades y desconocimiento estatal en diferentes aspectos: desde el reconocimiento del docente como pilar en el proceso de enseñanza-aprendizaje, el hacinamiento de los estudiantes en las escuelas y el recurrente en establecimientos universitarios de orden público; esa realidad se legitima en la cobertura, a tal punto de negar una educación crítica y contextualizada articulada a la realidad desde la eficiencia y con lógicas de inmediatez empresarial, como fue el caso de Chile, en 2011, con la instauración de la Ley de Calidad y Equidad de la Educación:

(...) - en enero de este año 2011 -, en acuerdo nuevamente con gran parte de los senadores y diputados de la Concertación, que entrega mayor poder y recursos a los sostenedores municipales para administrar los establecimientos, lo que consolida la 
municipalización de la educación, al mismo tiempo que permite la flexibilización absoluta del Estatuto Docente, precarizando la situación laboral del profesorado que trabaja en este sector (Budnik et al., 2011, 313)

En estas realidades, se correlacionan directamente la calidad, la cobertura y la financiación como aspectos básicos para que el Sistema educativo funcionara; sin embargo, su funcionamiento tiende a ser mediocre y no permite dimensionar los avances de cada uno de los aspectos; por tanto, la capacidad de cupos que un determinado plantel puede ofrecer no es símbolo de calidad, sino de masificación.

Las falencias no solamente se vislumbran en los municipios y ciudades, sino en la ineficacia estatal, pues su labor es nula o simplemente se reduce a transferir su responsabilidad a municipios y a la financiación privada; por esta razón, el aporte financiero hecho por el Estado se puede palpar en un simple "auxilio", que no alcanza a cubrir las necesidades básicas, no solamente de alimentación, sino, igualmente, las pedagógicas.

(...) la configuración de nuevas identidades docentes se realiza sobre un terreno de fuertes y crecientes desigualdades sociales, atravesado tanto por dinámicas fragmentadoras como por dinámicas centralizadoras (...) La impronta del ámbito de inserción sociocultural y la fragmentación socio económica creciente marcan esas brechas. (Birgin, 1999, 219)

Si bien la cobertura educativa ha ido mejorando significativamente en los niveles básicos - atención a la primera infancia - en países como México y Colombia, esto no es signo de calidad, como se lo ha reiterado antes; más bien, en particular, es un índice de desconocimiento y desatención a niveles como la educación media y superior, con lo cual se puede inferir que la educación que se imparte estaría por debajo de los estándares exigidos por entes internacionales, pues la calidad exigida no se cumple y aquí la cobertura no es sinónimo de calidad. Al respecto, Márquez Jiménez menciona que

la población adulta de nuestro país mantiene un bajo perfil educativo (8.6 años promedio de escolaridad) que contrasta ampliamente con el nivel alcanzado en los países desarrollados (el promedio de años de escolaridad entre los países de OCDE es de 11.3 años). $(2012,110)$ 
Resulta claro que, de mantenerse esta condición, no se tendrán muchas oportunidades de ser competitivos en la economía globalizada y la sociedad del conocimiento.

Así, se está ante una educación que dice responder a discursos internacionales, porque ni desde su propio contexto respeta el derecho a la educación y la calidad; más bien su funcionamiento corresponde a una educación discriminatoria y desigual, en que no se piensa en la población con menos recursos económicos; es decir, una educación pobre para pobres que, en primer lugar, no paga lo justo a los maestros, que no se responsabiliza de la dotación, ni de los servicios de los planteles; segundo, que contribuye con una suma sujeta a una serie de parámetros arbitrarios, que justifican en una cruda crisis fiscal que se fortaleció desde la descentralización administrativa, donde los impuestos llegan de la población de recursos bajos y la de los altos se encubre por su firme posición social; tercero, implanta modelos cosmopolitas para presentar avances respecto a la comunidad extranjera y, con esto, encajar resultados no tan buenos y tendientes a mejorar en pruebas europeas, como PISA. En ese orden de ideas, se puede afirmar que en:

(...) la institucionalización y la existencia de un proceso de fragmentación, todos estos segmentos están totalmente asociados al cambio del lugar del estado, a la aparición del mercado como el que dicta la lógica de organización del sistema y a la profundización de la sociedad de consumo. Todos estos procesos tienen que ver con el impacto cultural sobre el sistema educativo que tienen estos cambios en la estructura de la sociedad (Tiramonti, 2005, 104)

Es posible decir, además, que Colombia y varios países latinoamericanos — caso paralelo de México — han dado respuesta a parámetros impulsados por organismos multilaterales, como el Banco Mundial, la OCDE y el Banco Interamericano de Desarrollo, y a raíz de esto han reformado y orientado sus políticas educativas públicas actuales, que se establecen vertiginosamente y atienden a nuevas lógicas educativas, que transforman el sentido y la forma de asumir la educación.

\section{Conclusiones}

Se necesita reflexionar en la educación como un bien común, del cual la sociedad igualitariamente tuviera beneficios, donde, por la intervención del Estado, se pueda acceder a una educación sin brechas económicas ni sociales; no obstante, esta labor masificadora se produce de la manera menos idílica posible, 
pues resulta perentorio reconocer, también, que se encuentra en predisposición con el sector privado, que abona o reviste determinados porcentajes para la entrada de la sociedad a la educación.

De modo que estos porcentajes de ninguna manera deben interferir con la función estatal, que debe situarse en pro del bien común y no de un bien de mercado. Frecuentemente, se promueve una educación como bien de mercado y no como una oportunidad de formación de conocimiento, lo que sitúa a los individuos como capital humano con capacidad de moverse ágilmente y seguir la eficiencia y eficacia en el amplio espacio mercantilista, para proveerlo de ganancias materiales; es decir, la educación se piensa para el desarrollo económico de mercado y no desde una mirada igualitaria en pro del crecimiento profesional de las mayorías, pues se une estrechamente a factores determinantes sobre cómo influye y qué tipo de educación se requiere, desde las exigencias capitalistas para el desarrollo de los países. Por lo tanto, se puede inferir que la mentalidad capitalista se liga a la educación, lo que no resulta beneficioso para el crecimiento humano de estos países en vía de desarrollo, pues concibe una política que compromete los recursos públicos y las realidades contextuales de todo un Sistema educativo y, consecuentemente, de una sociedad.

En ese sentido, es necesaria una educación igualitaria, que entienda y explore los contextos propios de las comunidades, ya que en América Latina no se puede seguir replicando, sin previa consulta, dictámenes internacionales, pues sus características culturales, económicas políticas y sociales la tornan diferente y diversa para reducirse a un régimen evaluativo y pedagógico masificador, que no permite que se piense la generación propia de conocimiento.

De ahí, se tiene que re-pensar el papel de la educación como derecho, en un proceso de lucha en el terreno educativo, la financiación estatal, la reivindicación de la autonomía y la democratización de los espacios educativos, para afrontar la crisis de valores que la acompaña, por la preponderancia de la lógica económica, para proponer otro proyecto de educación, que no fuera una mera reproducción de Europa para las periferias, y se plantease un tipo de enseñanza no mercantil y, a su vez, unos Planes de estudio basados en las propias necesidades y en la recuperación del pensamiento latinoamericano. 


\section{Referencias}

Álvarez Gallego, Alejandro. «Los límites de la economía de la educación: abrir la mirada». Revista colombiana de educación. No. 67 (2014): 19-46.

Birgin, Alejandra. El trabajo de enseñar. Entre la vocación y el mercado: las nuevas reglas del juego. Buenos Aires: Troquel, 1999.

Braslavsky, Cecilia y Acosta, Felicitas. El estado de la enseñanza de la formación en gestión y política educativa en América Latina. Buenos Aires: Unesco/ IIEP, 2001.

Bresser-Pereira, Luiz Carlos. "Estado y mercado en el nuevo desarrollismo". Nueva Sociedad. No. 210 (2007): 110-125.

Budnik, Jenny Assaél et al. "La empresa educativa chilena". Educación y Sociedad. Vol. 32. No. 115 (2011): 305-322.

Cabrera, Francisco. "La prioridad no concretada. Financiamiento de la educación en América Latina y el Caribe". La Piragua - Revista Latinoamericana de Educación y Política. Vol. 2. No. 26 (2007): 1-8.

Centro de Análisis de Políticas Públicas, México Evalúa. Diez puntos para entender el gasto educativo en México. Consideraciones sobre su eficiencia. México: Centro de Análisis de Políticas Públicas/México Evalúa, 2011. $1-20$.

De Moura Castro, Claudio e Ioschpe, Gustavo. La remuneración de los maestros en América Latina ¿es baja? ¿Afecta la calidad de la enseñanza? Buenos Aires: Preal, 2007.

Feldfeber, Myriam. "La regulación de la formación y el trabajo docente: un análisis crítico de la "Agenda Educativa" en América Latina». Educación y Sociedad. Vol. 28. No. 99 (2007): 444-465.

Galindo, Camila Andrea et al. "Repercusión del proyecto neoliberal en la Educación Superior". AGO.USB. Vol. 15. No. 1 (2015): 73-94. 
González, Ivonne et al. Panorama Fiscal de América Latina y el Caribe: La movilización de recursos para el financiamiento del desarrollo sostenible. Santiago de Chile: ONU, 2017.

Kleiman, Ariel. "Economía de la educación: reseña de enfoques". Estudios políticos. No. 25 (2000): 147-165.

Leyva López, Soraya y Cárdenas Almagro, Antonio. "Economía de la educación: capital humano y rendimiento educativo". Análisis Económico. Vol. 17. No. 36 (2002): 79-106.

Mallama Martínez, Gabriel. "Gestión educativa en Colombia: un análisis desde la política educativa pública". Trabajo de grado de Especialización en Gerencia Social de la Educación, Universidad Pedagógica Nacional, 2014.

Márquez Jiménez, Alejandro. "El financiamiento de la educación en México: Problemas y alternativas". Perfiles educativos. Vol. 34. No. especial (2012): 107-117.

Martínez Rodríguez, Francisco Miguel y Amador Muñoz, Luis V. "Educación y desarrollo socio- económico. Contextos educativos. No. 13 (2010): 83-97.

Miñana Blasco, Carlos y Rodríguez, José Gregorio. La educación en el contexto neoliberal. http://www.humanas.unal.edu.co/red/files/3112/7248/4191/ Artculos-eduneoliberal.pdf

Morduchowicz, Alejandro y Duro, Luisa. La inversión educativa en América Latina y el Caribe. Las demandas de financiamiento y asignación de recursos. Buenos Aires: IIPE/Unesco, 2007.

Morduchowicz, Alejandro. Discusiones de la economía de la educación. Buenos Aires: Losada, 2004.

Organización de las Naciones Unidas para la Educación, la Ciencia y la Cultura/ Comisión Económica para América Latina. Financiamiento y gestión de la educación en América Latina y el Caribe. San Juan, Puerto Rico: Cepal, 2004, 1- 27. 
Papa, Gabriel. "La herencia del neoliberalismo en América Latina y los márgenes de maniobra de los gobiernos progresistas". Análisis y propuestas. Montevideo: Friedrich Ebert Stiftung, 2004. 1-24.

Saforcada, Fernanda y Vassiliades, Alejandro. "Las leyes de educación en los comienzos del siglo XXI: del neoliberalismo al postconsenso de Washington en América del Sur”. Educación y Sociedad. Vol. 32. No. 115 (2011): 287 -304 .

Tiramonti, Guillermina. "La trama de la desigualdad educativa". Diálogos Pedagógicos. No. 5 (2005): $94-109$.

Tomasevski, Katarina. "Indicadores del derecho a la educación". Revista Instituto Interamericano de Derechos Humanos. Vol. 40. No. 40 (2004): 341-388.

Vega Cantor, Renán. "Contrarreforma educativa en Colombia”. El Ágora USB. Vol. 11. No. 2 (2011): 335-379. 